\title{
Synthese special issue introduction
}

\author{
Alex Malpass · Chris Gifford
}

Received: 6 April 2012 / Accepted: 6 April 2012 / Published online: 8 June 2012

(C) Springer Science+Business Media B.V. 2012

This special issue of Synthese is dedicated to the notion of branching-time (BT). We have contributions from several leading figures in the field (namely Nuel Belnap, Thomas Müller and Tomasz Placek), and from several younger researchers (Matt Farr, Alex Malpass, Jacek Wawer and Alastair Wilson). The issue is a selected collection of papers, most of which given at a conference held in Bristol in August 2010. Although it was not originally given at the conference itself, we are delighted to be able to present Belnap's paper, Newtonian determinism to branching space-times indeterminism in two moves which was originally published by Mentis Verlag in 2007. Part of the reason for the present collection is to mark the 20 year milestone from the publication of Belnap's Branching Spacetime (1992) paper (originally published in Synthese).

Branching-time was originally a theory about the semantics of modal logics which contained the resources to express both temporal and modal notions. It is well known that the idea was first suggested to Arthur Prior by the young Saul Kripke in a letter, dated September 3rd 1958. Kripke's idea was that one could represent the future in an "indetermined system" as a tree of ever branching possibilities. Starting at a particular point in the tree, one faces certain incompatible future possibilities. This inevitably means that, at a later time, some branches which used to be accessible have become inaccessible, representing the philosophical and existential truth that as time passes certain things become impossible. This interaction between time and modality fascinated Prior, and by the time of his next major book-length publication (Past, Present and Future) 9 years later, we find him using Kripke's model as a way of formalising answers to the problem of future contingents (or Divine Foreknowledge) associated

\footnotetext{
A. Malpass $(\varangle) \cdot$ C. Gifford

University of Bristol, Bristol, UK

e-mail: Alex.Malpass@bristol.ac.uk

C. Gifford

e-mail: cg6577@bristol.ac.uk
} 
with William of Ockham and C. S. Peirce. These methods are still being developed today, the Ockhamist by Belnap, and the Peircean by Alberto Zanardo, amongst others.

The next great milestone in the history of BT comes some 25 years later with the publication of Belnap's Branching Spacetime paper, and the creation of the theory of the same name (BST). BST exhibits both a spatio-temporal aspect and an indeterministic "modal" aspect of alternative possible historical courses of events. An essential feature of BST is that it can also represent spatial or space-like relationships as part of its (more or less) relativistic theory of spatio-temporal relations; this ability is essential for the representation of local (in contrast with "global") indeterminism.

In his essay in this collection, Belnap indicates how BST might be seen to grow out of Newton's deterministic and non-relativistic theory by two independent moves: (1) Taking account of indeterminism, and (2) attending to spatio-temporal relationships in a spirit derived from Einstein's theory of special relativity. Since (1) and (2) are independent, one can see that there is room for four theories: Newtonian determinism, branching time indeterminism, relativistic determinism, and (finally) branching space-times indeterminism. This is an important paper, as it constitutes the only short and easily accessible introduction to BST written by the original creator of the theory.

Placek's paper in the collection represents an important development to the theory of BST. The paper sketches a concept of individuals within BST, and thus lays some crucial groundwork in the program of connecting the austere language of Belnap's original theory to the more familiar setting of agents facing choices in an indeterministic world. Placek connects the debate up to major themes in contemporary analytic philosophy by discussing Kripkean modal intuitions concerning individuation and necessity, and Lewis's objections to branching individuals.

In Müller's fascinating paper, we get a unique overview of the notion of branching, taken in its most general sense. He traces the metaphor of 'branching' through some of its uses and argues that the metaphor works the same way in physics as in philosophy. He describes a number of different notions of possibility, thereby sketching a 'landscape of possibilities'. In the final section of the paper he looks at the place of branching-based possibilities in that larger landscape of possibilities. His message is that far from being an outlandish metaphysical extravagancy, branching-based possibilities are epistemically as well as metaphysically basic.

In his paper, Wilson assesses the prospects for combining contemporary Everettian quantum mechanics (EQM) with branching-time semantics. He begins by outlining the salient features of 'decoherence-based' EQM, and of the 'consistent histories' formalism that is often used in conceptual discussions of EQM. This formalism permits of both 'branching worlds' and 'parallel worlds' interpretations; Wilson argues that the metaphysics of EQM is in this sense underdetermined by the physics. He discusses prominent arguments by Lewis and Belnap both for and against the notion of branching, and relates it to debates in EQM over the notion of objective probability. His conclusion is that Everettians are better off without branching-time semantics.

Farr's paper addresses the relationship between branching spacetime theories and metaphysical issues concerning the direction of time. His first concern is Storrs McCall's 'dynamic' account of BT which features such controversial metaphysical theses as 'branch-attrition'. He argues in a similar vein to Smart and Nerlich that these features require a model with two temporal dimensions, which he uses to address 
alleged conceptual problems with McCall's model. This model, Farr argues, illustrates that the flow of time and branch attrition are distinct concepts. In the second half of the paper, Farr examines the 'no backward branching' (NBB) feature of BST and the time-asymmetric modal account of indeterminism it entails. He argues that independent justifications offered for NBB either implicitly presuppose the relevant time asymmetry, or are inconclusive.

In the final paper, Malpass \& Wawer present a development of the Thin Red Line (TRL) theory. The central idea of the TRL is that there is such a thing as the 'actual future', even in the presence of indeterminism. This theory is widely held to suffer from various serious problems, many of which are articulated by Belnap et al. In their paper, Malpass \& Wawer propose several new TRL semantics, each with differing degrees of success, leading up to their final semantics, which is a cross between the TRL and supervaluationism.

We are happy to present such a wide and varied set of papers, some of which develop well established theories, and some of which question the very foundations of those theories themselves. We believe that this is good evidence that BT is, 54 years after it was first suggested by Kripke, a thriving environment in which to do analytical philosophy. 